\title{
Physiotherapy in early phase of low back pain
}

\author{
This article was published in the following Dove Press journal: \\ Orthopedic Research and Reviews \\ 17 March 20I I \\ Number of times this article has been viewed
}

\section{Markku Paatelma \\ Department of Education, Auron - OMT Center, \\ Helsinki, Finland}

Correspondence: Markku Paatelma

Tallinnanaukio IB, Fin-00930

Helsinki, Finland

Tel +358405014822

Fax +35893239250

Email markku.paatelma@auron.fi

\begin{abstract}
Low back pain (LBP) is a substantial health problem and has subsequently attracted a considerable amount of research both in the early and chronic phases. Chronic, nonspecific LBP indicates limited effectiveness from most commonly applied interventions and approaches, but it seems to be opposite in the early phase. Intervention is more effective than advice on staying active in acute LBP, leading to more rapid improvement in function, mood, quality of life, and general health. We compared physiotherapy (PT) that involved 3-7 treatment sessions based on subclassification in early phase LBP (acute and subacute LBP lasting $<3$ months) to one session of PT that advised staying active, in 134 LBP patients. Low back and leg pain, disability, and days of sick-leave were evaluated. After 12 months, all groups had only minimal pain and disability. In the advice-only group, those patients who had radiating pain had less improvement compared with other groups, and increasing days of sick-leave because of LBP after 12 months. Compared with the advice-only group, orthopedic manual therapy and McKenzie methods seemed to be slightly more effective than one session of assessment in pain and disability.
\end{abstract}

Keywords: OMT, advice, low back pain

\section{Introduction}

Low back pain (LBP) is a substantial health problem and has subsequently attracted a considerable amount of research both in the early and chronic phase. Clinical trials evaluating the efficacy of a variety of interventions for chronic nonspecific LBP indicate limited effectiveness for most commonly applied interventions and approaches. ${ }^{1}$ It seems to be opposite in the early phase. Gellhorn et $\mathrm{al}^{2}$ have shown that there is a lower risk of subsequent medical service usage among patients who received physiotherapy (PT) early after an episode of acute LBP relative to those who received PT at later times. According to Wand et $\mathrm{al}^{3}$, intervention is more effective than advice on staying active in acute LBP, leading to more rapid improvement in function, mood, quality of life, and general health. The timing of intervention affects the development of psychosocial features. ${ }^{3}$ In a systematic review of randomized controlled trials (RCTs) on advice for the management of LBP, the authors concluded: "advice to stay active is sufficient for acute LBP". ${ }^{4}$ No conclusions could be drawn as to the content and frequency of advice that is most effective for subacute LBP, due to the limited number and poor quality of RCTs. For chronic LBP, there is strong evidence to support the use of advice to remain active in addition to specific advice relating to the most appropriate exercise, and/or functional activities to promote active self-management. 
Classifying patients with nonspecific LBP into meaningful subgroups is thought to provide assistance for clinical management, and to increase the power of outcomes assessments, and has been targeted as an important research priority. The importance of classifying patients into homogeneous subgroups has been emphasized, and has been said to be one of the biggest challenges in physiotherapy. ${ }^{5}$ Use of homogeneous subgroups is considered by many experts to be essential for the improvement of clinical trials related to patient management and clinical outcomes. ${ }^{5-10}$

We compared PT that involved 3-7 treatment sessions based on subclassification in early phase LBP (acute and subacute LBP lasting $<3$ months) to one session of PT that advised staying active.

\section{Methods}

\section{Participants}

Participants ( $\mathrm{N}=134)$ were selected according to the following inclusion criteria: working-aged, employed people with current nonspecific LBP with or without radiating pain to lower leg; patients who visited their occupational physicians 0-7 days after their last LBP episode and had started the first treatment on day 8 or later; and the back pain episode could be the first or recurrent. Exclusion criteria were: pregnancy, low back surgery less than 2 months previously, and 'red flags' that indicate serious spinal pathology. ${ }^{11}$ Patients were randomized into three groups: 45 allocated to orthopedic manual therapy (OMT), 52 to McKenzie (or Mechanical Diagnosis and Therapy [MDT]), and 37 to the advice-only group $^{12}$ (Table 1).

In both treatment groups, the physical therapists treated their subjects independently by the method in which they were certified. All treatments were provided to each individual participant by the same therapist. The OMT was carried out by a physical therapist with 20 years of clinical experience in this field. The McKenzie method was carried out by a physical therapist with 10 years of experience in this therapy method. The physical therapist who advised the subjects to stay active and continue normal daily living had 5 years of clinical experience in treating patients with LBP.

All the participants provided written informed consent before the study, and the study protocol was approved by the local ethics committee.

A power calculation determined that the study was slightly underpowered (needing 50 subjects/group).

\section{PT}

\section{OMT group}

In the OMT group, the participants were clinically assessed and classified into the five patho-anatomical and patho-mechanical subgroups. ${ }^{13}$ The group underwent pain treatment, specific mobilization or stabilization, spinal manipulation if indicated, and muscle-stretching techniques. ${ }^{14-16}$ Typically, between three and five individually selected home exercises were prescribed to actively stabilize or mobilize the lower back, and stretching exercises were to be performed once a day.

\section{The McKenzie group}

In the McKenzie group, the participants were clinically assessed and classified into the three mechanical syndromes. ${ }^{17}$ If a syndrome was present, then one of the treatment principles of mechanical therapy was selected as the management strategy. This consisted of an educational component,

Table I Baseline demographics of I34 patients allocated to receive OMT or McKenzie therapy or advice-only

\begin{tabular}{|c|c|c|c|}
\hline & OMT $(n=45)$ & $\operatorname{McK}(n=52)$ & Advice-only $(n=37)$ \\
\hline \multicolumn{4}{|l|}{ Age } \\
\hline Mean (SD) & $44(10)$ & $44(9)$ & $44(15)$ \\
\hline \multicolumn{4}{|l|}{ Gender } \\
\hline Females/males (\%) & $41 / 59$ & $31 / 69$ & $35 / 55$ \\
\hline \multicolumn{4}{|l|}{ History of present LBP episode } \\
\hline Acute: $0-6$ weeks (\%) & 48 & 56 & 50 \\
\hline Subacute: from 7 weeks to 3 months (\%) & 52 & 44 & 50 \\
\hline On sick-leave because of LBP (\%) & 16 & 17 & 8 \\
\hline \multicolumn{4}{|l|}{ History of LBP } \\
\hline $0-5$ episodes $(\%)$ & 54 & 52 & 54 \\
\hline$>6$ episodes $(\%)$ & 46 & 48 & 46 \\
\hline \multicolumn{4}{|l|}{ Symptom localization } \\
\hline LBP only (\%) & 29 & 19 & 31 \\
\hline Radiating pain (\%) & 71 & 81 & 69 \\
\hline
\end{tabular}

Abbreviations: LBP, low back pain; McK, McKenzie method; OMT, orthopedic manual therapy; SD, standard deviation. 
supported with the book, and an active therapy component that provided instructions on exercises repeated several times a day according to the principles of the approach. ${ }^{18-20}$

\section{Advice-only group}

Participants in the advice-only group received 45-60 minutes counseling from a physiotherapist concerning good prognosis for LBP, pain tolerance, medication, and early return to work. For support, a two-page educational back booklet was also supplied. ${ }^{21}$ The patients in this group were told to avoid bed rest and advised to continue their routine as actively as possible, including exercise activities, within the limits permitted by their back pain.

\section{Measurements}

\section{Low back and radiating pain}

A visual analog scale (VAS) allowed the participant to rate current intensity of radiating leg and LBP from 0 to $100 .^{22}$

\section{Disability}

A 0-24-point scale Roland-Morris Disability Questionnaire allowed measurement of disability in daily activities in relation to LBP in the previous 3 months. ${ }^{23}$

\section{Sick-leave because of LBP}

During the 3-, 6-, and 12-month follow-up points, participants were asked whether they had taken sick-leave for current LBP, and the number of sick-leave days after the last follow-up.

\section{Effect sizes}

Comparison of treatment effects between all groups was analyzed, calculating effect sizes according to the following categories: effect size of $0.2-0.3$ to be a "small" effect, around 0.5 a "medium" effect, and $>0.8$, a "large" effect. $^{23}$

\section{Data analysis}

The means and differences between LBP patients in three different groups were calculated. For the significance in the difference between the groups, Student's $t$-tests for parametric distribution, and Mann-Whitney $U$ test for nonparametric distribution were used. ${ }^{25}$

\section{Results}

Treatment effects were compared between two PT approaches (OMT and McKenzie) and one counseling approach (advice). The absolute values of pain and disability indices and percentages of patients in sick-leave because of LBP at the 3-, 6-, or 12-month follow-up are shown in Table 2.

\section{LBP (VAS)}

At the 3-month follow-up point, back pain (VAS) decreased in all groups $12-21 \mathrm{~mm}$ from baseline, but there was no treatment effect when comparing therapy groups to the adviceonly group. A small effect size (ES) of 0.3 was seen in the McKenzie compared with the advice-only group. After the

Table 2 Outcome measures at baseline, 3-, 6-, and I2-month follow-up visits

\begin{tabular}{|c|c|c|c|}
\hline & OMT $(n=45)$ & $\operatorname{McK}(n=52)$ & Advice-only $(n=37)$ \\
\hline \multicolumn{4}{|l|}{ Baseline values } \\
\hline Leg pain (VAS, mm) ${ }^{\mathrm{a}}$ & $20(26.2)$ & $16(25.4)$ & $16(21.6)$ \\
\hline LBP (VAS, mm) ${ }^{\mathrm{a}}$ & $35(19.5)$ & $32(19.7)$ & $37(2.8)$ \\
\hline Roland-Morris $(0-24)^{b}$ & $9(5.7)$ & $9(4.6)$ & $8(4.1)$ \\
\hline Sick-leave because of LBP (\%) & 16 & 17 & 8 \\
\hline \multicolumn{4}{|l|}{ Outcome measures at 3 mo } \\
\hline Leg pain (VAS, mm) ${ }^{\mathrm{a}}$ & $6(18.2)$ & I (8.4) & $4(12.9)$ \\
\hline LBP (VAS, mm) ${ }^{\mathrm{a}}$ & $18(16.6)$ & $10(15.4)$ & 17 (17.9) \\
\hline Roland-Morris $(0-24)^{b}$ & $2(4.9)$ & I (4.0) & $0(4.2)$ \\
\hline Sick-leave because of LBP (\%) & 5 & 26 & II \\
\hline \multicolumn{4}{|l|}{ Outcome measures at 6 mo } \\
\hline Leg pain (VAS, mm) ${ }^{\mathrm{a}}$ & $4(15.1)$ & I (7.I) & $8(18.5)$ \\
\hline LBP (VAS, mm) ${ }^{\mathrm{a}}$ & $14(17.8)$ & $10(10.3)$ & $22(20.2)$ \\
\hline Roland-Morris $(0-24)^{b}$ & I (3.8) & $0(1.8)$ & I (4.9) \\
\hline Sick-leave because of LBP (\%) & 6 & 7 & 19 \\
\hline \multicolumn{4}{|l|}{ Outcome measures at $12 \mathrm{mo}$} \\
\hline Leg pain (VAS, mm) ${ }^{\mathrm{a}}$ & $2(17.6)$ & $0(10.5)$ & $8(16.7)$ \\
\hline LBP (VAS, mm) ${ }^{\mathrm{a}}$ & II (I7.3) & $8(19.4)$ & $16(16.1)$ \\
\hline Roland-Morris $(0-24)^{\mathrm{b}}$ & $0(3.7)$ & I (2.I) & $0(4.5)$ \\
\hline Sick-leave because of LBP (\%) & 3 & 4 & 23 \\
\hline
\end{tabular}

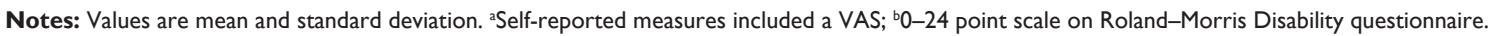
Abbreviations: LBP, low back pain; McK, McKenzie method; mo, months; OMT, orthopedic manual therapy; VAS, visual analog scale. 
6-month follow-up, back pain (VAS) decreased 20-24 mm in the therapy groups, and $4 \mathrm{~mm}$ in the advice-only group from baseline. There was a medium ES between the OMT and the advice-only group (0.42), and the McKenzie and advice-only group (0.76). At the 12-month follow-up, back pain (VAS) decreased in all groups 14-22 $\mathrm{mm}$ from baseline. Small ESs were found in the OMT (0.23) and in the McKenzie (0.17) groups compared with the advice-only group. Decrease of LBP at the 12-month follow-up was as follows: OMT 60\%, McKenzie 59\%, and advice-only 45\% (Figure 1, Table 3).

\section{Leg pain (VAS)}

At the 3-month follow-up point, leg pain (VAS) decreased in all groups $12-15 \mathrm{~mm}$ from baseline. A small ES of 0.3 was seen in the McKenzie compared with the advice-only group. At the 6-month follow-up, leg pain decreased 15-16 $\mathrm{mm}$ in the therapy groups and $8 \mathrm{~mm}$ in the advice-only group from baseline. A medium ES was seen in the McKenzie (0.6) and in the OMT group (0.5) compared with the advice-only group. At the 12-month follow-up, leg pain was decreased $16-18 \mathrm{~mm}$ in the therapy groups and $8 \mathrm{~mm}$ in the advice-only group from baseline. A small ES was seen in the McKenzie $(0.4)$ and in the OMT (0.3) groups compared with the adviceonly group. Decrease of radiating leg pain at 12-month follow up was as follows: OMT 74\%, McKenzie 75\%, and advice-only $26 \%$ (Figure 1 , Table 3 ).

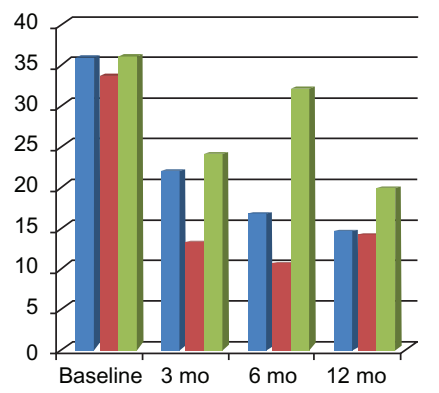

$$
\text { Low back pain (VAS) }
$$$$
\text { OMT }
$$$$
\text { Low back pain (VAS) }
$$$$
\text { McK }
$$

Low back pain (VAS)

Advice

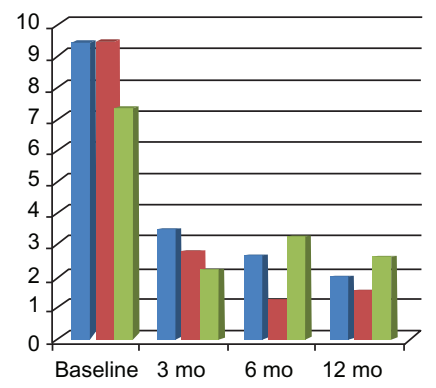

Disability (R-M) OMT

- Disability (R-M) McK

nisability (R-M) Advice

\section{Roland-Morris (R-M) disability}

At the 3-month follow-up point, R-M disability was decreased in all groups 7-8 points. A small ES of 0.2 was seen in the McKenzie and in the OMT group compared with the adviceonly group. At the 6-month follow-up, disability decreased in the therapy groups $8-9$ points and 7 points in the advice-only group from baseline. A medium ES of 0.5 was seen in the OMT group compared with the advice-only group, and also in the McKenzie group compared with the advice-only group (ES: 0.7). At 12-month follow-up, disability was decreased in all groups 5-8 points from baseline. A medium ES of 0.8 was seen in the McKenzie group and in the OMT group (0.6) compared with the advice-only group. Decrease of disability index was at 12-month follow-up as follows: OMT 80\%, McKenzie 85\%, and advice-only 64\% (Figure 1, Table 3).

\section{Sick-leave (days)}

At the 3-month follow-up point, days of sick-leave were decreased in the OMT group by 3 days, there was no change in the advice-only group, but there was an increase of 8 days in the McKenzie group from baseline. A medium ES of 0.6 was seen in the OMT group compared with the advice-only group, and an ES of 0.5 in the advice-only group compared with the McKenzie group. Change in days of sick-leave from baseline in the OMT group was -3 days and in the McKenzie group +8 days compared with the advice-only group. At the 6-month follow-up, days of sick-leave were
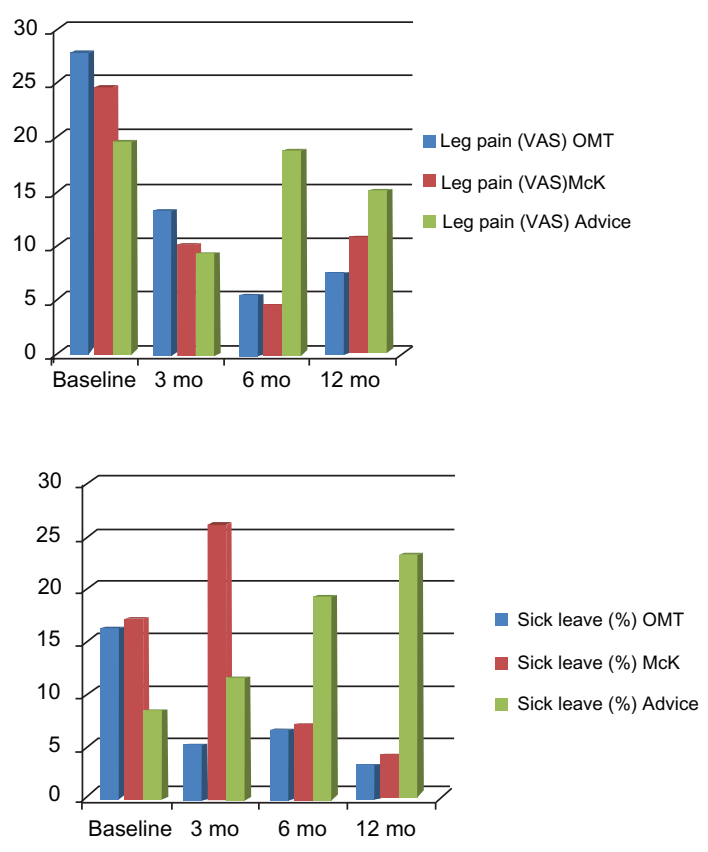

Figure I Low back pain (VAS), leg pain (VAS), disability (R-M), and sick leave (\%).

Abbreviations: McK, McKenzie method; mo, months; OMT, orthopedic manual therapy; R-M, Roland-Morris; VAS, visual analog scale. 
Table 3 The effect sizes between therapy groups (OMT and McK) compared with the advice-only group

\begin{tabular}{|c|c|c|c|}
\hline Low back pain & Effect size & Leg pain & Effect size \\
\hline OMT vs Advice 3 mo & 0.04 & OMT vs Advice 3 mo & 0.15 \\
\hline OMT vs Advice 6 mo & 0.42 & OMT vs Advice 6 mo & 0.52 \\
\hline OMT vs Advice 12 mo & 0.23 & OMT vs Advice $12 \mathrm{mo}$ & 0.35 \\
\hline McK vs Advice 3 mo & 0.30 & McK vs Advice 3 mo & 0.34 \\
\hline McK vs Advice 6 mo & 0.76 & McK vs Advice 6 mo & 0.61 \\
\hline McK vs Advice $12 \mathrm{mo}$ & 0.17 & McK vs Advice 12 mo & $0.4 I$ \\
\hline Disability & & Disability & \\
\hline OMT vs Advice 3 mo & 0.17 & OMT vs Advice 3 mo & 0.50 \\
\hline OMT vs Advice 6 mo & 0.54 & OMT vs Advice 6 mo & 0.53 \\
\hline OMT vs Advice 12 mo & 0.59 & OMT vs Advice 12 mo & 0.35 \\
\hline McK vs Advice 3 mo & 0.17 & McK vs Advice 3 mo & -0.62 \\
\hline McK vs Advice 6 mo & 0.73 & McK vs Advice 6 mo & 0.52 \\
\hline McK vs Advice $12 \mathrm{mo}$ & 0.79 & McK vs Advice 12 mo & 0.11 \\
\hline
\end{tabular}

Note: Effect size of $0.2-0.3$ is a "small" effect, around 0.5 a "medium" effect, and $\geq 0.8$, a "large" effect. ${ }^{24}$

Abbreviations: McK, McKenzie method; mo, months; OMT, orthopedic manual therapy.

decreased in the OMT group by 3 days, and 2 days in the McKenzie, and increased 1 day in advice-only group, from baseline. A medium ES (0.6) was seen in the OMT group and in McKenzie group compared with the advice-only group. Change in days of sick-leave from baseline in the OMT group was -4 days and in the McKenzie group -3 days compared with the advice-only group. At the 12-month follow-up, days of sick-leave were decreased in the OMT group by 3 days, but there was an increase of 1 day in the McKenzie and 4 days in the advice-only group, from baseline. A small ES (0.4) was seen in the OMT group compared with the advice-only group. Change in days of sick-leave from baseline in the OMT group was -7 days and in the McKenzie group -3 days compared with the advice-only group (Figure 1, Table 3).

Decrease in back pain (VAS) in 1-year follow-up was more than $20 \mathrm{~mm}$ in all groups. Decrease in leg pain (VAS) was in the OMT group $18 \mathrm{~mm}$ and $16 \mathrm{~mm}$ in the McKenzie groups, and $8 \mathrm{~mm}$ in the advice-only group. The minimally clinically important change (MCIC) for pain on VAS should be close to $20 \mathrm{~mm} \cdot{ }^{26,27}$

Decrease in R-M disability was 9 points in the OMT group, 8 points in the McKenzie group, and 8 points in the advice-only group. The MCIC for functional disability measured with R-M should be at least 3.5 points. ${ }^{26,27}$

At baseline, there were fewer days of sick-leave because of LBP in the advice-only group. But during treatment periods and 12-months follow-up there were more days of sick-leave in the McKenzie group compared with the OMT group, especially at the 3-month follow-up point $(P=0.004)$. Despite the increasing percentage of patients in sick-leave at 6- and 12-months follow-up in the advice-only group, the difference compared with other groups was not statistically significant $(P=0.03)$.

\section{Discussion}

At baseline, subjects in three subgroups did not differ from each other in disability or in low back or leg pain. Despite, half the patients having had more than six LBP episodes (Table 1), recurrences were few in the treatment groups during the 1-year of follow-up. Our results are in line with similar pragmatic studies. ${ }^{28-32}$ We showed the effectiveness of OMT and McKenzie type of PT during at least one year follow-up.

When comparing the effects of OMT and McKenzie with one counseling session with a physiotherapist (advice-only) on low back pain/leg pain, disability, and sick-leave, no significant differences emerged between the OMT and McKenzie groups in pain and disability scores at any follow-up point, except in sick-leave. Compared with the advice-only group, OMT and McKenzie methods seemed to be slightly more effective than was one session of assessment in pain and disability. This difference was seen usually at the 6-month follow-up point.

After 12 months, all groups had only minimal pain and disability. In the advice-only group, those patients who had radiating pain (leg VAS) had less improvement compared with other groups, and increasing days of sick-leave because of LBP after 12 months.

Advice to stay active has for a long time been recommended as best practice in international evidence-based guidelines for the management of acute LBP, despite there being some conflicting evidence. ${ }^{33}$ Malmivaara et $\mathrm{al}^{34}$ concluded that among patients with acute LBP, continuing ordinary activities within the limits permitted by the pain leads to more rapid recovery than either bed rest or back-mobilizing exercises. The McKenzie method and chiropractic manipulation had shown similar effects 
and costs, and patients receiving these treatments had only marginally better outcomes than those receiving the minimal intervention of advice on acute LBP. ${ }^{28}$ Our results confirm that advice-only treatment seems to be effective in the first 3 months for back and leg pain, and in disability. The other two groups showed the effectiveness up to 12 months in these parameters. We did not evaluate cost-effectiveness of treatments. But increase of sick-leave days in the 3-months follow-up in the McKenzie group, which was statistically significant and increasing days of sick-leave from 3-months to 12-months follow-up in the advice-only group, should be considered when planning new studies.

Pengel et $\mathrm{al}^{35}$ reported that for participants with subacute LBP, physiotherapist-directed exercise and advice were each slightly more effective than placebo. The effect was greatest when the interventions were combined. Storheim and Grotle ${ }^{36}$ made the same conclusion, that a combination of exercise and advice was slightly better than placebo for subacute LBP. In our study, exercises and advice were part of the treatment strategy in every group.

Given that the effectiveness of treatment for subacute symptoms will directly influence the development of chronicity, these results would suggest that education and awareness of the causes and consequences of back pain may be a valuable treatment component for this patient subgroup. ${ }^{4}$ Education and cognitive components were also included in our therapies.

\section{Conclusion}

All groups in our study improved in back and radiating leg pain, and in dysfunction, in a way which is clinically significant. Improvement was seen during the first 3 months in all groups, and improvement increased in the therapy groups also in the 6- and 12-months follow-up, which could not be seen in the advice-only group. Despite there being no statistically significant differences between the advice-only group and the other two groups, the differences could be regarded as clinically significant. An interesting factor was the lower adherence of the advice-only group to self-treatment or unwillingness to participate in follow-up visits, so in future, the adherence to self-treatment should be studied.

Small but maybe important differences between the therapy groups and the advice-only group in radiating pain and disability favor the OMT or McKenzie-type approach in the early phase of LBP. These results can also be an encouragement to study further how the inexpensive adviceonly method could be developed in the early phase of LBP. A small number of days of sick-leave will reflect the low risk of the OMT-type approach, not only during the treatment sessions, but also during the 1-year follow-up, with a patient self-care program.

\section{Disclosure}

The author reports no conflicts of interest in this work.

\section{References}

1. Wand BM, O'Connell NE. Chronic non-specific low back pain-sub-groups or a single mechanism? BMC Musculoskelet Disord. 2008;9:11.

2. Gellhorn AC, Chan L, Martin B, Friedly J. Management patterns in acute low back pain: the role of physical therapy. Spine. Epub 2010 Nov 19.

3. Wand BM, Bird C, McAuley JH, Doré CJ, MacDowell M, De Souza LH. Early intervention for the management of acute low back pain: a singleblind randomized controlled trial of biopsychosocial education, manual therapy, and exercise. Spine. 2004;29(21):2350-2356.

4. Liddle SD, Gracey JH, Baxter GD. Advice for the management of low back pain: a systematic review of randomised controlled trials. Man Ther. 2007;12(4):310-327.

5. Dankaerts W, O'sullivan PB, Straker LM, Burnett AF, Skouen JS. The inter-examiner reliability of a classification method for non-specific chronic low back pain patients with motor control impairment. Man Ther. 2006;11(1):28-39.

6. Delitto A, Erhard RE, Bowling RW. A treatment-based classification approach to low back syndrome: identifying and staging patients for conservative treatment. Phys Ther. 1995;75(6):470-485.

7. Fritz JM, George S. The use of a classification approach to identify subgroups of patients with acute low back pain. Interrater reliability and short-term treatment outcomes. Spine. 2000;25(1):106-114.

8. Werneke MW, Hart DL. Categorizing patients with occupational low back pain by use of the Quebec Task Force Classification system versus pain pattern classification procedures: discriminant and predictive validity. Phys Ther. 2004;84(3):243-254.

9. Hall H, McIntosh G, Boyle C. Effectiveness of a low back pain classification system. Spine. 2009;9:648-657.

10. Dankaerts W, O’Sullivan P, Burnett A, Straker L, Davey P, Gupta R. Discriminating healthy controls and two clinical subgroups of nonspecific chronic low back pain patients using trunk muscle activation and lumbosacral kinematics of postures and movements: a statistical classification model. Spine. 2009;34:1610-1618.

11. Bigos S, et al. Acute Low Back Problems in Adults. Clinical Practice Guideline, Agency for Health Care Policy and Research, AHCPR Publication No.95-0643, 1994.

12. Paatelma M, Kilpikoski S, Simonen R, Heinonen A, Alen M, Videman T. Orthopaedic manual therapy, McKenzie method or advice only for low back pain in working adults: a randomized controlled trial with one year follow-up. J Rehabil Med. 2008;40(10): 858-863.

13. Paatelma M, Karvonen E, Heinonen A. Inter-tester reliability in classifying acute and subacute low back pain patients into clinical subgroups: a comparison of specialists and non-specialists. a pilot study. J Man Manip Ther. 2009;17(4):221-229.

14. Krauss J, Evjenth O, Creighton D. Translatoric Spinal Manipulation. Minneapolis, USA: Lakeview Media LLC; 2006.

15. Kaltenborn FM, Evjenth O, Kaltenborn TB, Morgan D, editors. Manual Mobilization of the Joints. The Kaltenborn Method of Joint Examination and Treatment, valid. The Spine. 4th ed. Norli Bokhandel: Oslo, Norway; 2003.

16. Evjenth O, Hamberg J. Autostretching. Sweden: Alfta Rehab Förlag; 1989.

17. Kilpikoski S, Airaksinen O, Kankaanpaa M, Leminen P, Videman T, Alen M. Interexaminer reliability of low back pain assessment using the McKenzie method. Spine. 2002;27(8):E207-E214. 
18. McKenzie R. Treat Your Own Back. Waikanae, New Zealand: Spinal Publication Ltd; 1985.

19. Udermann B, Spratt KF, Donelson R, Mayer J, Graves J. Can a patient educational book change behaviour and reduce pain in chronic low back pain patients? Spine. 2004;4:425-435.

20. McKenzie R, May S. The Lumbar Spine Mechanical Diagnosis and Therapy. Waikanae, New Zealand: Spinal Publication Ltd; 2003.

21. Burton A, Waddell G, Tillotson K, Summerton N. Information and advice to patients with back pain can have a positive effect. A randomized controlled trial of a novel educational booklet in primary care Spine. 1999;24:2484-2491.

22. Scott J, Huskisson E. Vertical and horizontal visual analogue scales. Ann Rheum Dis. 1978;38:560.

23. Roland M, Fairbank J. The Roland-Morris Disability Questionnaire and the Ostwestry Disability Questionnaire. Spine. 2000;25:3115-3124.

24. Cohen J. A power primer. Psychol Bull. 1992;112:155-159.

25. Portney L, Watkins M. Foundation of Clinical Research: Applications to Practice. NJ: Prentice Hall Health; 2000.

26. Ostelo RW, de Vet HC. Clinically important outcomes in low back pain. Best Pract Res Clin Rheumatol. 2005;19:593-607.

27. Kovacs FM, Abraira V, Royuela A, et al. Minimal clinically important change for pain intensity and disability in patients with nonspecific low back pain. Spine. 2007;32:2915-2920.

28. Cherkin DC, Deyo RA, Battié M, Street J, Barlow W. A comparison of physical therapy, chiropractic manipulation, and provision of an educational booklet for the treatment of patients with low back pain. N Engl J Med. 1998;339:1021-1029.
29. Brealey S, Burton K, Coulton S, et al. UK Back pain Exercise And Manipulation (UK BEAM) trial - national randomised trial of physical treatments for back pain in primary care: objectives, design and interventions. BMC Health Serv Res. 2003;3(1):16.

30. Cairns MC, Foster NE, Wright C. Randomized controlled trial of specific spinal stabilization exercises and conventional physiotherapy for recurrent low back pain. Spine. 2006;31:E670-E681.

31. Ferreira PH, Ferreira ML, Maher CG, Herbert RD, Refshauge K. Specific stabilisation exercise for spinal and pelvic pain: a systematic review. Aust J Physiother. 2006;52(2):79-88.

32. Niemisto L, Rissanen P, Sarna S, Lahtinen-Suopanki T, Lindgren KA, Hurri H. Cost-effectiveness of combined manipulation, stabilizing exercises, and physician consultation compared to physician consultation alone for chronic low back pain: a prospective randomized trial with 2-year follow-up. Spine. 2005;30:1109-1115.

33. Williams CM, Maher CG, Hancock MJ, et al. Low back pain and best practice care: a survey of general practice physicians. Arch Intern Med. 2010;170:271-277.

34. Malmivaara A, Häkkinen U, Aro T, et al. The treatment of acute low back pain - bed rest, exercises, or ordinary activity? $N$ Engl J Med. 1995;332:351-355.

35. Pengel LH, Refshauge KM, Maher CG, Nicholas MK, Herbert RD, McNair P. Physiotherapist-directed exercise, advice, or both for subacute low back pain: a randomized trial. Ann Intern Med. 2007;146:787-796.

36. Storheim K, Grotle M. Combination of exercise and advice was slightly better than placebo for subacute low back pain. Aust J Physiother. 2007;53:286.
Orthopedic Research and Reviews

\section{Publish your work in this journal}

Orthopedic Research and Reviews is an international, peer-reviewed, open access journal focuses on the patho-physiology of the musculoskeletal system, trauma, surgery and other corrective interventions to restore mobility and function. Advances in new technologies, materials, techniques and pharmacological agents are particularly welcome. The journal welcomes

\section{Dovepress}

original research, clinical studies, reviews \& evaluations, expert opinion and commentary, case reports and extended reports. The manuscript management system is completely online and includes a very quick and fair peer-review system, which is all easy to use. Visit http://www.dovepress. com/testimonials.php to read real quotes from published authors. 